\title{
Degrees of Freedom of Multi-Cell MI MO Interference Broadcast Channels With Distributed Base Stations
}

\author{
Hongbing Huang ${ }^{1}$, Junyi Liu ${ }^{1}$, Yi Zhang ${ }^{1}$, Qing Cai ${ }^{1}$, Bowei Zhang ${ }^{2}$, Fengwen Jiang ${ }^{2}$ \\ ${ }^{1}$ State Grid Zhejiang Electric Power Co., Ltd Information \& Telecommunication Branch \\ Zhejiang, China, 310026 \\ [e-mail: \{james_hb, shshzaa, zhangyi_zjdl, 18204070632\}@163.com] \\ ${ }^{2}$ College of Communication Engineering, Chongqing University \\ Chongqing, China, 400044 \\ [e-mail: boevyzbw@163.com, fengwj@cqu.edu.cn] \\ *Corresponding author: Bowei Zhang
}

Received March 22, 2018; revised May 11, 2018; accepted September 8, 2018;

published February 28, 2019

\begin{abstract}
In this paper, we investigate the degrees of freedom (DoF) of a multi-cell multi-user multiple-input multiple-output (MIMO) interference broadcast channel (IBC) with non-cooperation distributed base stations (BS), where each BS serves users of its corresponding cell. When all BSs simultaneously transmit their own signals over the same frequency band in the MIMO IBC, the edge users in each cell will suffer the inter-cell interference (ICI) and inter-user interference (IUI) signals. In order to eliminate the ICI and IUI signals, a distributed space time interference alignment (DSTIA) approach is proposed where each BS has only limited access to distributed moderately-delay channel state information at the transmitter (CSIT). It is shown that the DSTIA scheme can obtain the appreciate DoF gains. In addition, the DoF upper bound is asymptotically achievable as the number of antenna at each BS increases. It is shown that the DSTIA method can get DoF gains over other interference alignment schemes with delayed CSIT in literature. Moreover, the DSTIA method can attain higher DoFs than the IA schemes with global CSIT for certain antenna configurations.
\end{abstract}


Keywords: Degrees of freedom, interference broadcast channel, channel state information, multiple-input multiple-output, interference alignment

\section{Introduction}

Interference alignment (IA), which was first introduced in [1], is a representative interference management approach to effectively eliminate interference in wireless interference networks. The main idea of IA is aligning interference signals into lower signal space dimensions so that each receiver can obtain more interference-free signal space dimensions. The authors in [2] enlarged the idea of IA method to a $K$-user interference channel and demonstrated that the degrees of freedom (DoF) of $K / 2$ can be obtained. DoF is a useful metric in characterizing the capacity of wireless networks.

\subsection{Related work}

Since the traditional IA technology needs global channel state information (CSI) at the transmitter (CSIT) to construct precoding matrices, distributed nature of the users and delayed CSIT are major barriers to preform IA. In order to overcome the feedback delay problem, Maddah-Ali et al. proposed a retrospective interference alignment (RIA) and showed that the global delayed CSIT can also be utilized to increase the DoF [3]. Subsequently, global delayed CSIT was exploited in a variety of interference networks [4-7].

Obtaining global CSIT is another bottleneck among distributed transmitters. To address this problem, Lee et al. proposed the space time interference alignment (STIA) method by using the local moderately-delayed CSIT [8-9] in the independent and identically distributed (i.i.d.) block fading channels. In [9-10], the authors show that the local moderately-delayed CSIT can effectively increase the DoF in the multiple-input single-output (MISO) broadcast channel and multiple-input multiple-output (MIMO) X channel, respectively.

IA is a still useful method to reduce inter-cell interference (ICI) and inter-user interference (IUI) signals dimensions in cellular interference networks [11]-[19]. The authors in [12] used the grouping IA method and showed that the optimal DoF is achievable in the MIMO two-cell two-user interference broadcast channel (IBC). The grouping IA scheme was further extended to MIMO multi-cell IBC in [18]. In [24], the authors show that the quality of the desirable link can be enhanced based on bidirectional relaying protocol and successive interference cancelation technique for the two-cell network with a two-way relay. The authors in [19] proposed the IA method based on linear beamforming strategies in the MIMO IBC with arbitrary number of users per cell. In [23], the authors study the multi-cell system 
with a multi-antenna two-way relay and show that the quality of signal reception can be improved based on the optimum relay precoder. In [22], the authors show that the irregular antenna arrays can maximize achievable rate in massive MIMO systems.

It is worth noting that the above DoF results in [11]-[19] are obtained under a requirement of global CSIT. In practical scenarios, it is unreasonable to ignore the impact of feedback delay. Recently, the authors in [20] first exploited the delayed CSIT to obtain a higher DoF than the time division multiple access (TDMA) scheme for the two-cell MIMO cellular interference network. The authors in [21] applied the RIA scheme in [3] to the two-cell two-user MIMO IBC and showed that sum-DoF can be promoted by using the delayed CSIT. The existed IA schemes with two cells cannot directly be extended to multi-cell multi-user IBC. The achievable DoF is still unknown for the MIMO multi-cell multi-user cellular interference network. Hence, we need to explore whether local CSIT can increase DoF gains in the MIMO multi-cell multi-user IBC with non-cooperation distributed base stations (BS). Another interesting question is that whether the delayed and current CSIT can also be utilized to align both ICI and IUI signals? We would like to address the problem in this paper.

\subsection{Main contributions}

We aim to investigate the obtained DoF of the multi-cell multi-user IBC with non-cooperation distributed BSs, where each BS serves users of its corresponding cell. When all BSs simultaneously transmit their own signals over the same frequency band in the MIMO IBC, the edge users in this scenario will receive both of ICI and IUI signals. The key for obtaining higher DoFs is jointly exploit the overheard ICI and IUI information at each user when there is only local moderately-delayed CSIT at each BS. For this purpose, we propose a distributed space time interference alignment (DSTIA) method to investigate the obtained DoF of the MIMO IBC. With the help of local moderately-delayed CSIT, the proposed approach can align both of ICI and IUI signals over the timeline of the past and current interference patterns, thereby providing more interference-free signal space dimensions at each user. Our main contributions in this work are listed as follows:

1. We first analyze the transmission mode of the two-cell two-user MIMO IBC with distributed and moderately-delayed CSIT. With the proposed DSTIA scheme, we obtain the new achievable DoF for the two-cell two-user MIMO IBC. Then, we extend the proposed DSTIA scheme to the $L$-cell $K$-user MIMO IBC. The DSTIA scheme performs in a distributed manner, yielding reduced feedback overhead for acquiring CSI.

2. We verify that the simulation results of the DSTIA scheme are consistent with theoretical DoF gains. we show that the proposed method can attain a higher DoF than the scheme in [8]. In addition, we analyze the sum-DoF performance of the DSTIA scheme when the number of antennas at BS increases gradually. The result implies that the DSTIA approach can asymptotically obtain the DoF upper bound when the number of antenna at 
each BS is large enough. Moreover, we compare the achievable DoFs among the DSTIA scheme, the RIA scheme in [21] and the IA schemes in [18] and [19], where local delayed CSIT, global delayed CSIT and global instantaneous CSIT are available respectively. Specifically, the proposed DSTIA approach can provide significant DoF gains over the RIA scheme [21], as well as a better performance compared to the IA schemes in [18] and [19] for certain antenna configurations. This implies that the strict condition of global CSIT is not necessarily required in some scenarios and can be gradually degraded.

This paper is organized as follows. In Section 2, we describe the system model. In Section 3, we introduce the DSTIA scheme and the achievable DoF of the MIMO IBC is investigated. The numerical simulations are presented in Section 4. In the end, this paper is concluded in Section 5 .

Notation: For any matrix $\mathbf{A}, \operatorname{rank}(\mathbf{A}), \mathbf{A}^{-1}$ and $\mathbf{A}^{*}$ denote the rank of $\mathbf{A}$, inverse and Hermitian transpose of $\mathbf{A}$, respectively.

\section{System model}

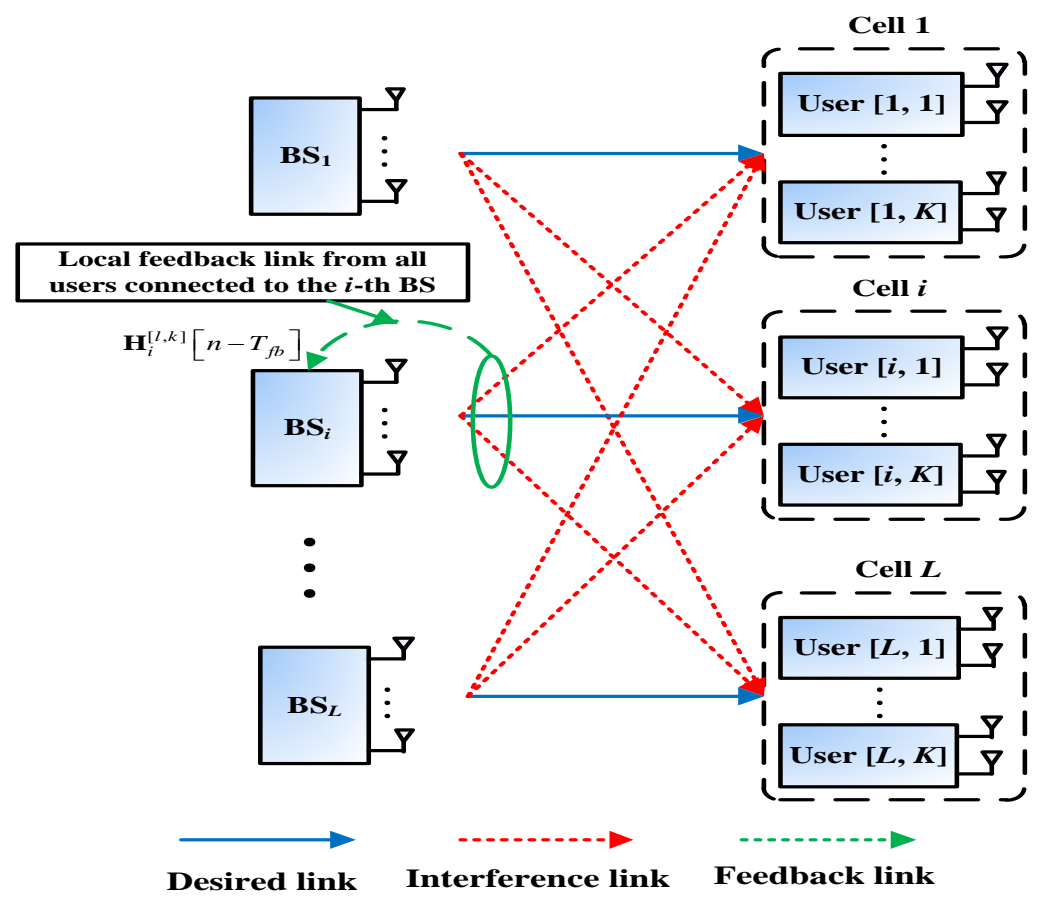

Fig. 1. The $L$-cell MIMO interfering broadcast channel

\subsection{Channel model}

We consider an $L$-cell MIMO IBC with $K(K \geq 2)$ users per cell in Fig.1, where each BS has $M$ antennas and each user has $N$ antennas. For notation convenience, we refer to the $k$-th user in the $l$-th cell as user $[l, k], l \in \boldsymbol{L}=\{1,2, \ldots, L\}$ and $k \in \boldsymbol{K}=\{1,2, \ldots, K\}$. In the considered 
channel, all nodes are located in the same frequency band and the $i$-th BS intends to send an independent message $W_{i}^{[i, k]}$ to its corresponding user $[i, k]$. When each BS simultaneously transmits data streams, each user will receive both of ICI and IUI signals. The received signal at the user $[l, k]$ is given by

$$
\mathbf{y}_{l, k}(n)=\sum_{i=1}^{L} \mathbf{H}_{i}^{[l, k]}(n) \mathbf{x}_{i}(n)+\mathbf{z}_{l, k}(n),
$$

where $\mathbf{H}_{i}^{[l, k]}(n)$ denotes $N \times M$ channel matrix from the $i$-th BS to the user $[l, k]$ at the $n$-th time slot, $\mathbf{x}_{i}(n)$ denotes the $M \times 1$ transmit signal vector at $i$-th $\mathrm{BS}, \mathbf{y}_{l, k}[n]$ denotes the $N \times 1$ received signal at the user $[l, k], \mathbf{z}_{l, k}(n)$ denotes the $N \times 1$ additive white Gaussian noise (AWGN) vector at the user $[l, k]$. It is assumed that each BS has only local CSI and the feedback link has $T_{f b}$ delay. In other words, the $i$-th BS can access to the local CSI $\mathbf{H}_{i}^{[l, k]}\left[n-T_{f b}\right]=\left\{\mathbf{H}_{i}^{[l, k]}(1), \mathbf{H}_{i}^{[l, k]}(2), \ldots, \mathbf{H}_{i}^{[l, k]}\left(n-T_{f b}\right)\right\}$ up to time slot $n$ for the user $[l, k]$. The channel is assumed to be an ideal block-fading channel, i.e., channel values are invariant for the channel coherence time $T_{C}$. The transmit power at each transmitter is assumed to be $P$, i.e., $\mathbf{E}\left(\left\|\mathbf{x}_{i}(n)\right\|^{2}\right) \leq P$. Each user can obtain an accurate estimate of its CSI. The noise terms are neglected in DoF analysis due to the fact that noise does not affect DoF.

\subsection{The CSI feedback model in block fading channels}

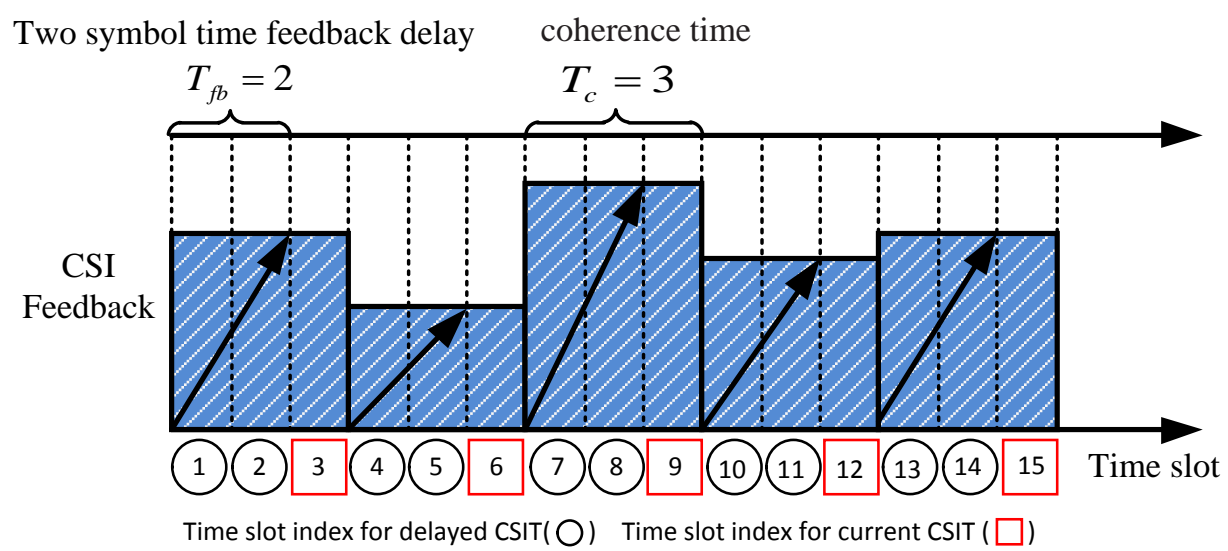

Fig. 2. Illustration of the CSI feedback in the block-fading channel

Following [8-10], each BS can obtain error-free but partially delayed CSI through feedback links. It is assumed that the channel coherence time is larger than the feedback delay, i.e., $T_{f b}<T_{C}$. Since the channel coefficient is constant in every channel block, each BS can obtain the current and delayed CSI. For instance, as illustrated in Fig. 2, we assume that the feedback delay $T_{f b}$ is equal to 2 and the channel coherence time is equal to 3. Each BS can 
obtain the current CSI in time slot 3, 6, 9, 12 and 15. To represent the delay characteristic of CSI, we define a parameter $\lambda$ called the normalized CSI feedback delay, i.e.,

$$
\lambda=\frac{T_{f b}}{T_{C}} .
$$

The case of $\lambda \geq 1$ means that the CSI is completely delayed. This paper considers the case of $0<\lambda<1$.

\subsection{Degrees of freedom}

According to [8-10], the achievable rate of each user depends on parameter $\lambda$ and signal-to-noise ratio (SNR). The achievable rate per time slot of the user $[i, k]$ over $n$ time slots can be written as

$$
R^{[i, k]}(\lambda, \mathrm{SNR})=\frac{\log _{2}\left|W_{i}^{[i, k]}(\lambda, \mathrm{SNR})\right|}{n} .
$$

The DoF of the user $[i, k]$ can be given by

$$
d^{[i, k]}=\frac{R^{[i, k]}(\lambda, \mathrm{SNR})}{\log _{2}(\mathrm{SNR})} .
$$

The sum-DoF of the $L$-cell MIMO IBC with $K$ users per cell is defined as

$$
\begin{aligned}
\mathrm{DoF}_{\text {sum }} & =\sum_{i=1}^{L} \sum_{k=1}^{K} d^{[i, k]} \\
& =\sum_{i=1}^{L} \sum_{k=1}^{K} \lim _{\mathrm{NRR} \rightarrow \infty} \frac{R^{[i, k]}(\lambda, \mathrm{SNR})}{\log _{2}(\mathrm{SNR})} .
\end{aligned}
$$

\section{The Proposed Distributed Space-Time Interference Alignment}

In this section, we explain the proposed DSTIA scheme in detail. For convenience, we denote $(L, M, N, K)$ for the $L$-cell MIMO IBC with $K(K \geq 2)$ users per cell, where each BS has $M$ antennas and each user is equipped with $N$ antennas.

\subsection{Motivating example}

We propose a DSTIA transmission scheme emphasizing the design of ICI and IUI alignment matrices at each BS. As a motivating example, we first consider a simple case with the system configuration $(L, M, N, K)=(2,6,2,2)$. In addition, we focus on the special case of $\lambda=\frac{T_{f b}}{T_{C}}=\frac{2}{3}$, i.e., each BS has access to current CSIT over two-thirds of the channel 
coherence time. Particularly, we select five time slots $n \in\{1,4,9,12,15\}$ for our DSTIA transmission scheme where the value of selected time slot belongs to a different channel block. Noteworthy, the transmitter has access to outdated CSIT in $n \in\{1,4\}$ and both of current and outdated CSIT in $n \in\{9,12,15\}$. In addition, we perform the TDMA scheme in other time slots as a time-sharing technique. We show that the sum-DoF of $24 / 5$ can be achieved, i.e., 24 independent data streams will be transmitted without interference over 5 time slots. The proposed DSTIA scheme involves two steps: redundancy transmission and interference alignment of ICI and IUI. We explain how each of these steps is performed to achieve the stated DoF.

1) Redundancy transmission

This step uses two time slots, i.e., $n \in\{1,4\}$. In time slot 1 , BS1 transmits the superposition of signals without precoding and BS 2 keeps silence. The transmit signal at the BS 1 is given by

$$
\mathbf{x}_{1}(1)=\mathbf{s}^{[1,1]}+\mathbf{s}^{[1,2]},
$$

where $\mathbf{s}^{[1,1]}=\left[s_{1}^{[1,1]} s_{2}^{[1,1]} \cdots s_{6}^{[1,1]}\right]^{T}$ and $\mathbf{s}^{[1,2]}=\left[s_{1}^{[1,2]} s_{2}^{[1,2]} \cdots s_{6}^{[1,2]}\right]^{T}$ denote the signal from BS 1 to user $[1,1]$ and user $[1,2]$, respectively. In time slot 1 , the received signal at each user is given by

$$
\mathbf{y}_{l, k}(1)=\mathbf{H}_{1}^{[l, k]}(1)\left(\mathbf{s}^{[1,1]}+\mathbf{s}^{[1,2]}\right), \quad l, k \in\{1,2\} .
$$

Note that $\mathbf{H}_{1}^{[l, k]}(1)$ is the $N \times M=2 \times 6$ channel matrix and $\mathbf{s}^{[1,1]}$ consists of 6 data streams. If we want to decode $\mathbf{s}^{[1,1]}$, we need to provide 6 interference-free linear independent equations. Moreover, although user [2,1] and user [2,2] only can receive the ICI signal in time slot 1 , the overheard ICI signal in time slot 1 is still useful to eliminate ICI signal in step two.

Similarly, in time slot 4, BS2 sends the superposition of signals without precoding and BS 1 keeps silence. The transmit signal at the BS 2 is given by

$$
\mathbf{x}_{2}(4)=\mathbf{s}^{[2,1]}+\mathbf{s}^{[2,2]},
$$

where $\mathbf{s}^{[2,1]}=\left[s_{1}^{[2,1]} s_{2}^{[2,1]} \cdots s_{6}^{[2,1]}\right]^{T}, \mathbf{s}^{[2,2]}=\left[s_{1}^{[2,2]} s_{2}^{[2,2]} \cdots s_{6}^{[2,2]}\right]^{T}$. In time slot 4 , the received 
signal at each user is given by

$$
\mathbf{y}_{l, k}(4)=\mathbf{H}_{2}^{[l, k]}(4)\left(\mathbf{s}^{[2,1]}+\mathbf{s}^{[2,2]}\right), \quad l, k \in\{1,2\} .
$$

The goal of the interference alignment step is to provide the 6 interference-free linear independent equations for each user.

2) Interference alignment of ICI and IUI.

This step uses three time slots, i.e., $n \in\{9,12,15\}$. In each time slot of this step, each BS sends a superposition of previously transmitted symbols by utilizing the linear beamforming matrix as

$$
\begin{array}{r}
\mathbf{x}_{1}(n)=\mathbf{V}^{[1,1]}(n) \mathbf{s}^{[1,1]}+\mathbf{V}^{[1,2]}(n) \mathbf{s}^{[1,2]}, n \in\{9,12,15\}, \\
\mathbf{x}_{2}(n)=\mathbf{V}^{[2,1]}(n) \mathbf{s}^{[2,1]}+\mathbf{V}^{[2,2]}(n) \mathbf{s}^{[2,2]}, n \in\{9,12,15\} .
\end{array}
$$

The received signal at each user is given by

$$
\begin{aligned}
\mathbf{y}_{l, k}(n)= & \mathbf{H}_{1}^{[l, k]}(n)\left(\mathbf{V}^{[1,1]}(n) \mathbf{s}^{[1,1]}+\mathbf{V}^{[1,2]}(n) \mathbf{s}^{[1,2]}\right) \\
& +\mathbf{H}_{2}^{[l, k]}(n)\left(\mathbf{V}^{[2,1]}(n) \mathbf{s}^{[2,1]}+\mathbf{V}^{[2,2]}(n) \mathbf{s}^{[2,2]}\right), n \in\{9,12,15\}
\end{aligned} .
$$

The central idea for constructing the precoding matrix is aligning the ICI and IUI signals in time slot $n \in\{1,4\}$ and $n \in\{9,12,15\}$. Therefore, BS 1 and BS 2 construct the precoding matrices $\mathbf{V}^{[1,1]}(n), \quad \mathbf{V}^{[1,2]}(n), \quad \mathbf{V}^{[2,1]}(n)$ and $\mathbf{V}^{[2,2]}(n)$ to satisfy

$$
\begin{aligned}
& \left\{\mathbf{H}_{1}^{[1,1]}(n) \mathbf{V}^{[1,2]}(n)=\mathbf{H}_{1}^{[1,1]}(1), \mathbf{H}_{2}^{[1,1]}(n) \mathbf{V}^{[2,1]}(n)=\mathbf{H}_{2}^{[1,1]}(4), \mathbf{H}_{2}^{[1,1]}(n) \mathbf{V}^{[2,2]}(n)=\mathbf{H}_{2}^{[1,1]}(4)\right\}, \\
& \left\{\mathbf{H}_{1}^{[1,2]}(n) \mathbf{V}^{[1,1]}(n)=\mathbf{H}_{1}^{[1,2]}(1), \mathbf{H}_{2}^{[1,2]}(n) \mathbf{V}^{[2,1]}(n)=\mathbf{H}_{2}^{[1,2]}(4), \mathbf{H}_{2}^{[1,2]}(n) \mathbf{V}^{[2,2]}(n)=\mathbf{H}_{2}^{[1,2]}(4)\right\}, \\
& \left\{\mathbf{H}_{1}^{[2,1]}(n) \mathbf{V}^{[1,1]}(n)=\mathbf{H}_{1}^{[2,1]}(1), \mathbf{H}_{1}^{[2,1]}(n) \mathbf{V}^{[1,2]}(n)=\mathbf{H}_{1}^{[2,1]}(1), \mathbf{H}_{2}^{[2,1]}(n) \mathbf{V}^{[2,2]}(n)=\mathbf{H}_{2}^{[2,1]}(4)\right\}, \\
\{ & \left.\mathbf{H}_{1}^{[2,2]}(n) \mathbf{V}^{[1,1]}(n)=\mathbf{H}_{1}^{[2,2]}(1), \mathbf{H}_{1}^{[2,2]}(n) \mathbf{V}^{[1,2]}(n)=\mathbf{H}_{1}^{[2,2]}(1), \mathbf{H}_{2}^{[2,2]}(n) \mathbf{V}^{[2,1]}(n)=\mathbf{H}_{2}^{[2,2]}(4)\right\},
\end{aligned}
$$

where $n \in\{9,12,15\}$ and (13),(14),(15) and (16) are designed for aligning IUI and ICI of user [1,1], user [1,2], user [2,1] and user [2,2], respectively. For example, we list the condition of $\mathbf{V}^{[1,2]}(n)$ as 


$$
\underbrace{\left[\begin{array}{l}
\mathbf{H}_{1}^{[1,1]}(n) \\
\mathbf{H}_{1}^{[2,1]}(n) \\
\mathbf{H}_{1}^{[2,2]}(n)
\end{array}\right]}_{\mathbf{G}=3 N \times M} \underbrace{\mathbf{V}^{[1,2]}[n]}_{M \times M}=\underbrace{\left[\begin{array}{c}
\mathbf{H}_{1}^{[1,1]}(1) \\
\mathbf{H}_{1}^{[2,1]}(1) \\
\mathbf{H}_{1}^{[2,2]}(1)
\end{array}\right]}_{\mathbf{F}=3 N \times M} .
$$

Since $3 N \times M=6 \times 6$ is a square matrix, the inverse matrix of $\mathbf{G}$ in (17) exists with probability one. Therefore, $\mathbf{V}^{[1,2]}(n)=\mathbf{G}^{-1} \mathbf{F}$. It is noteworthy that $\mathbf{V}^{[1,2]}(n)$ can also be found as the pseudo-inverse matrix of $\mathbf{G}$ exists, i.e., the condition of $M>3 N$ can be satisfied.

Similarly, $\quad \mathbf{V}^{[1,1]}(n), \quad \mathbf{V}^{[2,1]}(n)$ and $\mathbf{V}^{[2,2]}(n)$ can be found and (13), (14), (15) and (16) hold.

3) Decoding

For each user, it can eliminate all ICI and IUI signals and obtain 6 interference-free linear independent equations by performing interference cancellation, i.e., $\mathbf{y}_{l, k}(n)-\mathbf{y}_{l, k}(1)-\mathbf{y}_{l, k}(4), \quad n \in\{9,12,15\}$. For instance, we explain the decoding process for user $[1,1]$. Concatenating the received signals over 5 time slots, the equivalent input-output relationship at user $[1,1]$ is

$$
\underbrace{\left[\begin{array}{l}
\mathbf{y}_{1,1}(9)-\mathbf{y}_{1,1}(1)-\mathbf{y}_{1,1}(4) \\
\mathbf{y}_{1,1}(12)-\mathbf{y}_{1,1}(1)-\mathbf{y}_{1,1}(4) \\
\mathbf{y}_{1,1}(15)-\mathbf{y}_{1,1}(1)-\mathbf{y}_{1,1}(4)
\end{array}\right]}_{6 \times 1}=\underbrace{\left[\begin{array}{c}
\mathbf{H}_{1}^{[1,1]}(9) \mathbf{V}^{[1,1]}(9)-\mathbf{H}_{1}^{[1,1]}(1) \\
\mathbf{H}_{1}^{[1,1]}(12) \mathbf{V}^{[1,1]}(12)-\mathbf{H}_{1}^{[1,1]}(1) \\
\mathbf{H}_{1}^{[1,1]}(15) \mathbf{V}^{[1,1]}(15)-\mathbf{H}_{1}^{[1,1]}(1)
\end{array}\right]}_{\overline{\mathbf{H}}_{1}=6 \times 6} \mathbf{s}^{[1,1]} .
$$

Since all precoding matrices $\mathbf{V}^{[l, k]}(n)(n \in\{9,12,15\})$ are independently generated, the effective channel $\overline{\mathbf{H}}_{1}$ for user [1,1] has a full rank almost surely, i.e., $\operatorname{rank}\left(\overline{\mathbf{H}}_{1}\right)=6$. As a result, user [1,1] can decode 6 desired symbols in 5 time slots. In the same way, other users can decode 6 desired symbols in 3 time slots. As a result, 24 desired symbols can be transmitted in 5 time slots, which implies that the sum-DoF of 24/5 can be achieved. It is worth noting that the sum-DoF is calculated during five time slots $n \in\{1,4,9,12,15\}$. The main reason is that the TDMA scheme does not impact the achievable DoF of the DSTIA approach when the time slot $n \rightarrow \infty$. The detailed explanation will be presented in section 3.2 . 


\subsection{The general system}

The following theorem establishes the achievable DoF of the MIMO IBC with general system configuration.

Theorem 1: For the $L$-cell $K$-user MIMO IBC with moderately-delayed local CSIT, we can show that the following DoF is achievable:

$$
\mathrm{DoF}_{\text {sum }}=\frac{L K N A}{T_{\mathrm{IA}}}, M \geq(L-1) K N, A=\left\lfloor\frac{M}{N}\right\rfloor,
$$

where $T_{\mathrm{IA}}=A+L$ represents the number of time slots by using the proposed DSTIA method.

Proof: Without loss of generality, it is assumed that $Q+T_{\mathrm{IA}}-1$ channel blocks contain $T_{\mathrm{IA}}\left(Q+T_{\mathrm{IA}}-1\right)$ time slots. Therefore, each channel block contains $T_{\mathrm{IA}}$ time slots, i.e., $T_{C}=T_{\mathrm{IA}}$. The set of time slots $S_{t}=\left\{1,2, \ldots, T_{\mathrm{IA}}\left(Q+T_{\mathrm{IA}}-1\right)\right\}$ is defined to transmit data streams. Further,

we define $Q$ time slot sets $\left\{I_{1}, I_{2}, \ldots, I_{Q}\right\}$, each of which contains $T_{\mathrm{IA}}$ time slots, i.e., $I_{q}=\left\{t_{q, 1}, t_{q, 2}, \ldots, t_{q T_{\mathrm{IA}}}\right\}$, where $q \in\{1,2, \ldots, Q\}$. In the time slot $I_{q}$, we apply the DSTIA scheme. It is worth noting that each time slot of $I_{q}$ belongs to a different channel block. Then, the achievability of sum-DoF will be proved for each time slot set $I_{q}$. For simplicity, the index $l$ is omitted, i.e., $I_{q}=\left\{t_{1}, t_{2}, \ldots, t_{T_{\mathrm{IA}}}\right\}$. The proposed transmission scheme involves two steps.

1) Redundancy transmission

This step uses $L$ time slots, i.e., $n \in\left\{t_{1}, t_{2}, \ldots, t_{L}\right\}$. In time slot $t_{i}(i \in \boldsymbol{L})$, the $i$-th BS sends the following signal without precoding and all of the $j$-th ( $j \in \boldsymbol{L} \backslash i$ ) BSs keep silence.

The transmit signal at the $i$-th BS is given by

$$
\mathbf{X}_{i}\left(t_{i}\right)=\mathbf{s}^{[i, 1]}+\mathbf{s}^{[i, 2]}+\mathbf{s}^{[i, K]},
$$


where $\mathbf{s}^{[i, k]}=[s_{1}^{[i, k]}, S_{2}^{[i, k]}, \cdots, s_{N A}^{[i, k]}, \underbrace{0 \cdots 0}_{M-N A}\rfloor^{T}$. Since $A=\left\lfloor\frac{M}{N}\right\rfloor$ and the condition of $N A<M$ holds, each BS can utilize $M$ antennas to send NA data streams and $M-N A$ zero elements. In time slot $t_{i}(i \in \boldsymbol{L})$, the received signal at each user is given by

$$
\mathbf{y}_{l, k}\left(t_{i}\right)=\mathbf{H}_{i}^{[l, k]}\left(t_{i}\right)\left(\mathbf{s}^{[i, 1]}+\mathbf{s}^{[i, 2]}+\mathbf{s}^{[i, K]}\right)
$$

Note that $\mathbf{H}_{i}^{[l, k]}\left(t_{i}\right)$ is the $N \times M \quad(N<M)$ channel matrix and $\mathbf{s}^{[i, k]}$ consists of $N A$ data streams data streams. If we want to decode $\mathbf{s}^{[i, k]}$ at user $[i, k]$, we need to provide $N A$ interference-free linear independent equations for the data stream $\mathbf{s}^{[i, k]}$. At the end of step one, each user receives $K-1$ IUI signal vectors and $L(K-1)$ ICI signal vectors.

2) Interference alignment of ICI and IUI

This step takes $A$ time slots for providing $N A$ interference-free linear independent equations, i.e., $n \in\left\{t_{L+1}, t_{L+2}, \cdots, t_{A+L}\right\}$. In the time slot $n$, each BS sends a superposition of previously transmitted data streams by utilizing the linear beamforming matrix as

$$
\mathbf{x}_{i}(n)=\mathbf{V}^{[i, 1]}(n) \mathbf{s}^{[i, 1]}+\mathbf{V}^{[i, 2]}(n) \mathbf{s}^{[i, 2]}+\cdots+\mathbf{V}^{[i, K]}(n) \mathbf{s}^{[i, K]}, n \in\left\{t_{L+1}, t_{L+2}, \cdots, t_{A+L}\right\} .
$$

The received signal at each user is written as

$$
\mathbf{y}_{l, k}(n)=\sum_{i=1}^{L} \mathbf{H}_{i}^{[l, k]}(n)\left(\mathbf{V}^{[i, 1]}(n) \mathbf{s}^{[i, 1]}+\mathbf{V}^{[i, 2]}(n) \mathbf{s}^{[i, 2]}+\cdots+\mathbf{V}^{[i, K]}(n) \mathbf{s}^{[i, K]}\right), n \in\left\{t_{L+1}, t_{L+2}, \cdots, t_{A+L}\right\} .
$$

The key idea for constructing the precoding matrix $\mathbf{V}^{[l, k]}(n)$ is aligning the IUI signals in the $l$-th time slot and the ICI signals in the $j$-th $(j \in \boldsymbol{L} \backslash l)$ time slot with the IUI and ICI signals in time slots $n \in\left\{t_{L+1}, t_{L+2}, \cdots, t_{A+L}\right\}$ for user $[l, k]$. Then, we construct the precoding matrix $\mathbf{V}^{[l, k]}(n)$ as: 


$$
\underbrace{\left[\begin{array}{c}
\mathbf{H}_{l}^{[l, 1]}(n) \\
\vdots \\
\mathbf{H}_{l}^{[l, \bar{k}]}(n) \\
\mathbf{H}_{i}^{[l, 1]}(n) \\
\vdots \\
\mathbf{H}^{[l, K]}(n)
\end{array}\right]}_{\mathbf{C}=(L K-1) N \times M} \underbrace{\mathbf{V}^{[l, k]}(n)}_{M \times M}=\underbrace{\left[\begin{array}{c}
\mathbf{H}_{l}^{[l, 1]}\left(t_{l}\right) \\
\vdots \\
\mathbf{H}_{l}^{[l, \bar{k}]}\left(t_{l}\right) \\
\mathbf{H}_{i}^{[l, 1]}\left(t_{l}\right) \\
\vdots \\
\mathbf{H}_{i}^{[l, K]}\left(t_{l}\right)
\end{array}\right]}_{\mathbf{D}=(L K-1) N \times M}, \quad i, l \in \boldsymbol{L}, \quad k \in \boldsymbol{K}, \quad \bar{k} \in \boldsymbol{K} \backslash k .
$$

Since the condition of $M \geq(L K-1) N$ can be satisfied, the pseudo-inverse or inverse

matrix of $\mathbf{C}$ exists and $\mathbf{V}^{[l, k]}(n)$ exists with probability one.

3) Decoding

For each user, it can eliminate all ICI and IUI signals and obtain NA interference-free linear independent equations by performing interference cancellation, i.e., $\mathbf{y}_{l, k}(n)-\left(\sum_{i=1}^{L} \mathbf{y}_{l, k}\left(t_{i}\right)\right) \quad\left(n \in\left\{t_{L+1}, t_{L+2}, \cdots, t_{A+L}\right\}\right)$. For instance, we explain the decoding step for user [1,1] in detail. During time slots, the received signals at user [1,1] is written as

$$
\underbrace{\left[\begin{array}{c}
\mathbf{y}_{1,1}\left(t_{L+1}\right)-\left(\sum_{i=1}^{L} \mathbf{y}_{1,1}\left(t_{i}\right)\right) \\
\mathbf{y}_{1,1}\left(t_{L+2}\right)-\left(\sum_{i=1}^{L} \mathbf{y}_{1,1}\left(t_{i}\right)\right) \\
\vdots \\
\mathbf{y}_{1,1}\left(t_{A+L}\right)-\left(\sum_{i=1}^{L} \mathbf{y}_{1,1}\left(t_{i}\right)\right)
\end{array}\right]}_{\overline{\mathbf{Y}}=N A \times 1}=\underbrace{\left[\begin{array}{c}
\mathbf{H}_{1}^{[1,1]}\left(t_{L+1}\right) \mathbf{V}^{[1,1]}\left(t_{L+1}\right)-\mathbf{H}_{1}^{[1,1]}(1) \\
\mathbf{H}_{1}^{[1,1]}\left(t_{L+2}\right) \mathbf{V}^{[1,1]}\left(t_{L+2}\right)-\mathbf{H}_{1}^{[1,1]}(1) \\
\vdots \\
\mathbf{H}_{1}^{[1,1]}\left(t_{A+L}\right) \mathbf{V}^{[1,1]}\left(t_{A+L}\right)-\mathbf{H}_{1}^{[1,1]}(1)
\end{array}\right]}_{\overline{\mathbf{H}}=N A \times M} \mathbf{s}^{[1,1]}+\overline{\mathbf{Z}}_{1,1} .
$$

where $\overline{\mathbf{H}}$ denotes the equivalent channel matrix for user [1,1], $\overline{\mathbf{Z}}_{1,1}$ represents the equivalent noise at user [1,1]. It is worth noting that all precoding matrices $\mathbf{V}^{[l, k]}(n)$ ( $\left.n \in\left\{t_{L+1}, t_{L+2}, \cdots, t_{A+L}\right\}\right)$ are independently generated and $T_{\mathrm{IA}}=A+L$. Therefore, the rank of the effective channel $\overline{\mathbf{H}}$ is equal to $N A$, i.e., $\operatorname{rank}\left(\overline{\mathbf{H}}_{1}\right)=N A$. Therefore, user $[1,1]$ is able to decode $N A$ desired symbols in $T_{\text {IA }}$ time slots. In the same way, $N A$ desired symbols can be decoded at user $[l, k]$ during $T_{\mathrm{IA}}$ time slots. Consequently, a total of $L K N A$ desired symbols can be transmitted in $T_{\mathrm{IA}}$ time slots without interference, which implies that the 
$\frac{L K N A}{T_{\mathrm{IA}}}$ DoF is achieved. Hence, for the total time slot set $Q, \frac{L K N A Q}{T_{\mathrm{IA}} Q}$ DoF is achievable over $Q$ time slot sets.

Note that the total resource is $S_{t}=\left\{1,2, \ldots, T_{\mathrm{IA}}\left(Q+T_{\mathrm{IA}}-1\right)\right\}$. It is shown that $\frac{L K N A Q}{T_{\mathrm{IA}} Q}$ DoF can be obtained over $Q$ time slot sets ( $T_{\mathrm{IA}} Q$ time slots), i.e., $\left|I_{1} \cup I_{2} \ldots \cup I_{n}\right|=T_{\mathrm{IA}} Q$. For the remaining $T_{\mathrm{IA}}\left(T_{\mathrm{IA}}-1\right)$ time slots, we use a time-sharing technique between the DSTIA approach and the traditional TDMA method. By using the TDMA scheme, we can achieve additional $N T_{\mathrm{IA}}\left(T_{\mathrm{IA}}-1\right)$ DoF for the remaining $T_{\mathrm{IA}}\left(T_{\mathrm{IA}}-1\right)$ time slots. Therefore, the sum-DoF of this network can be given by

$$
\mathrm{DoF}_{\text {sum }}=\frac{L K N A Q+N T_{\mathrm{IA}}\left(T_{\mathrm{IA}}-1\right)}{T_{\mathrm{IA}} Q+T_{\mathrm{IA}}\left(T_{\mathrm{IA}}-1\right)} \stackrel{Q \rightarrow \infty}{=} \frac{L K N A}{T_{\mathrm{IA}}} .
$$

Consequently, as $Q$ goes to infinity, the sum-DoF can be achieved as $\frac{L K N A}{T_{\mathrm{IA}}}$. We thus complete the proof of Theorem 1.

Remark: Different from the scheme in [18, 19] with global CSIT, the beamforming matrix design of the proposed DSTIA scheme is only based on moderately-delayed local CSIT. Therefore, the proposed DSTIA scheme can reduce the amount of CSI feedback significantly. Furthermore, studying the DoF of the MIMO IBC with moderately-delayed local CSIT can provide a heuristic thought, where the delayed CSIT at each BS and the overheard interference information at each user can also be utilized to align both ICI and IUI signals.

\section{Numerical Results}

We demonstrate that the DSTIA scheme exactly attains the promised DoF obtained in Section 3. In order to explain the advantages of the proposed approach, the DSTIA scheme is compared with the traditional TDMA method, the IA approach with delayed CSIT in [21] and the IA schemes with global CSIT in [18] and [19]. The sum-rate simulation results are averaged over 10000 independent Monte Carlo runs.

We first explain how to calculate the sum rate of the MIMO IBC by applying the DSTIA method. Without loss of generality, we calculate $R^{[1,1]}$ as a representative. According to (25), 
the received signal at user $[1,1]$ is given by

$$
\overline{\mathbf{Y}}=\overline{\mathbf{H}} \mathbf{s}^{[1,1]}+\overline{\mathbf{Z}}_{1,1},
$$

where $\overline{\mathbf{H}}$ denotes the equivalent channel matrix for user [1,1], $\overline{\mathbf{Z}}_{1,1}$ represents the equivalent noise at user [1,1]. According to [8-10], the sum rate $R^{[1,1]}$ is written as

$$
R^{[1,1]}=\frac{\log _{2}\left[\operatorname{det}\left(\mathbf{I}+P \overline{\mathbf{H}} \mathbf{Z}^{-1} \overline{\mathbf{H}}^{*}\right)\right]}{T_{\text {IA }}},
$$

where $\mathbf{Z}=\mathbf{E}\left[\overline{\mathbf{Z}}_{1,1}\left(\overline{\mathbf{Z}}_{1,1}\right)^{*}\right]$, $\operatorname{det}[\cdot]$ and $\mathbf{E}[\cdot]$ denote the determinant and expectation operation, respectively. The sum rate $R^{[i, k]}(i \in \boldsymbol{L}, k \in \boldsymbol{K})$ can be computed in a similar way. The sum rate of this network is calculated by (29).

$$
R_{\mathrm{sum}}=\sum_{i=1}^{L} \sum_{k=1}^{K} R^{[i, k]} .
$$

Fig. 3 illustrates the sum rate of the DSTIA scheme according to different system configurations. The value of each system configuration is chosen according to Theorem 1 . The sum rate of the MIMO IBC is calculated by (29). It is worth noting that the slope value of the sum rate curve is equal to DoF/10lg2. It is shown that the slopes of the curves $(4,24,2,4),(5,40,2,5),(6,60,2,6)$ and $(7,84,2,7)$ are $24,40,60$ and 84 , respectively when SNR is high enough. This result shows that the simulation result is consistent with theoretical DoF in Theorem 1.

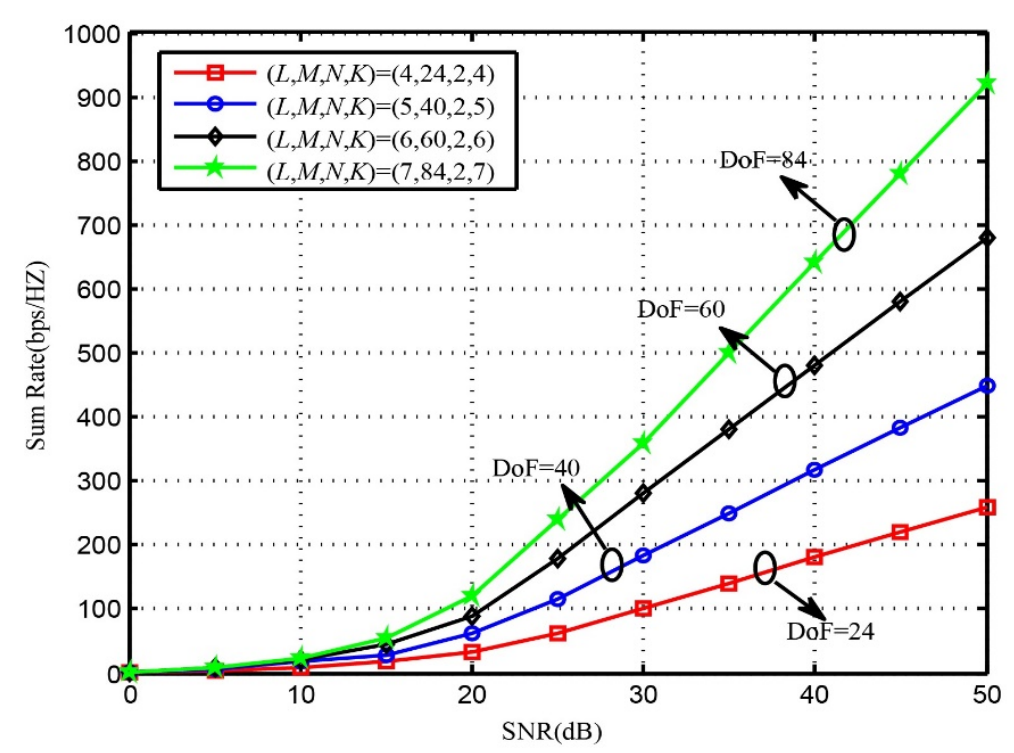

Fig. 3. The sum rate for the MIMO IBC with various antenna configurations 


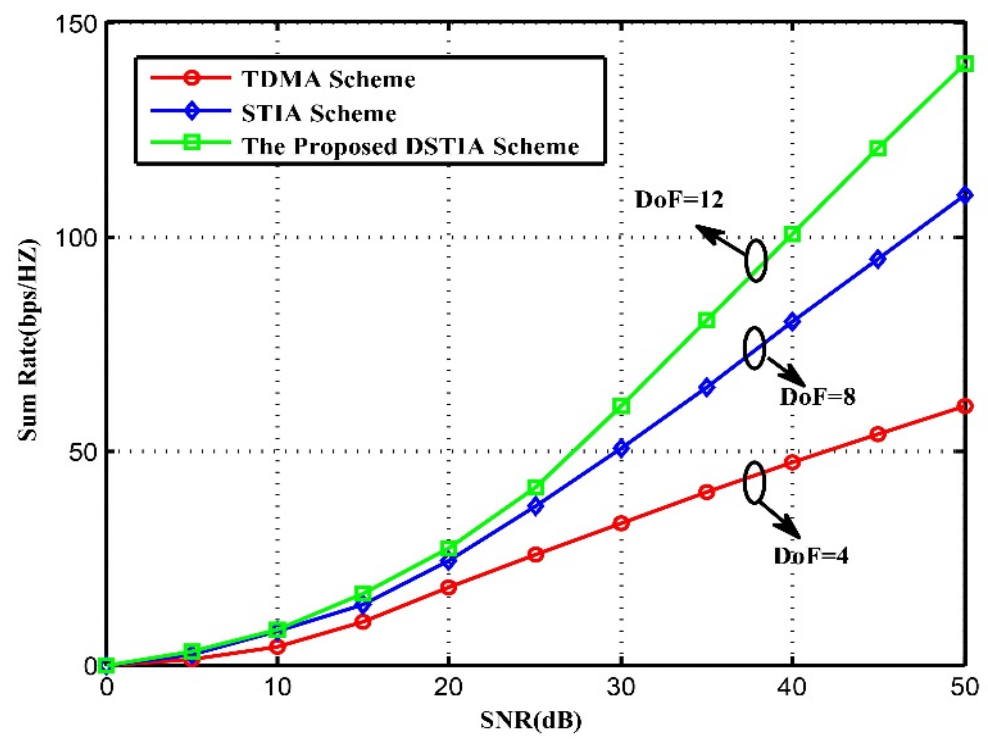

Fig. 4. Comparison of sum rate performance among different schemes under the same system configuration $(L, M, N, K)=(2,24,4,2)$

We compare the DSTIA scheme with traditional TDMA scheme and the STIA scheme in [8]. In order to avoid ICI and IUI signals, the conventional TDMA method needs $L K$ time slots to transmit streams. In each time slot, one of BSs transmits independent data streams to its corresponding user and the remaining BSs keep silence. Consequently, $N$ DoF can be trivially obtained by utilizing the TDMA scheme. The STIA scheme in [8] can utilize precoding technique to eliminate IUI signals in this network. The STIA scheme cannot align ICI signals and need $L$ time slots to send interference-free signals. Thus, the DoF of $K N$ can be obtained by using the STIA scheme. Fig. 4 shows the sum rate result, which compares the above three schemes under the same system configuration. It is illustrated that the proposed method provides about 200\% and 50\% DoF improvement compared to the TDMA scheme and the STIA method, respectively. The primary cause is that the proposed DSTIA scheme can use the delayed CSIT and the overheard interference information at BS to eliminate both ICI and IUI signals. 


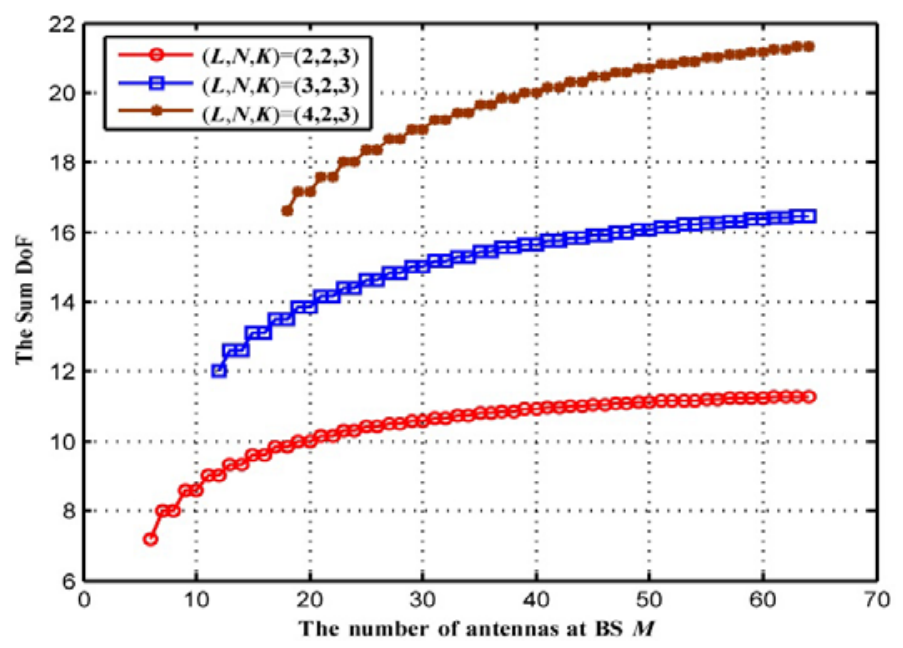

(a) The range of $M$ is $[6,64]$

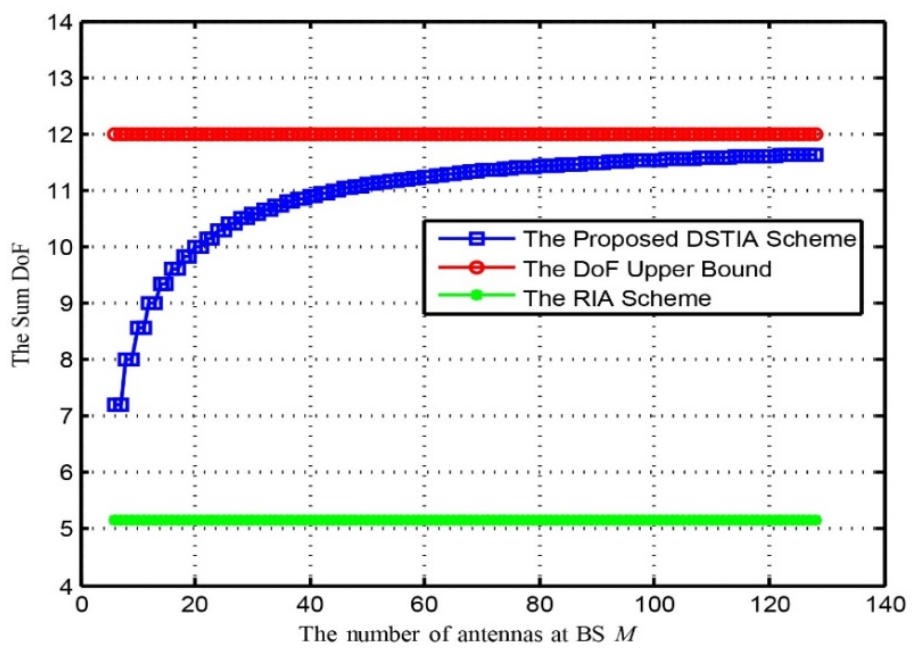

(b) The range of $M$ is $[6,128]$ and $(L, N, K)=(2,2,3)$

Fig. 5. The sum-DoF of the MIMO IBC

In Fig. 5, the sum-DoF of the MIMO IBC by using the proposed DSTIA scheme is given according to the number of antennas at BS $M$. In Fig. 5(a), we assume that $N=2, K=3, L$ and $M$ vary in the range of [2, 4] and [6, 64], respectively. It is shown that the sum-DoF increases gradually when the value of $M$ get larger. This implies that the proposed DSTIA scheme can utilize the signal space at each BS so that more ICI and IUI signals can be aligned into signal space at each user. However, it is worthy note that the sum-DoF does not increase at some specific values of $M$. For example, the sum-DoF does not increase when the value of $M$ 
varies from 19 to 20 for the curves of $(3,2,3)$ in Fig. 5(a). The main reason is that the obtained DoF is related to $\lfloor M / N\rfloor$ and the floor operation will keep the sum-DoF constant at some specific values of $M$. In Fig. 5(b), It is assumed that $(L, N, K)=(2,2,3)$ and the value of $M$ belongs to $[6,128]$. For comparison, we also consider the DoF upper bound and the RIA scheme in [21]. The RIA scheme can utilize the delayed CSIT to obtain 32/7 DoF. In the MIMO IBC with $N$ antennas per user, when the number of antennas at BS $M$ is larger than $K N$, each user can receive $N$ interference-free data streams at most in each time slot. Therefore, the DoF upper bound is $L K N$. It is shown that the achievable DoF of the RIA method is always 32/7 when the number of antennas at BS increases. The primary cause is that the RIA method cannot fully utilize signal spaces at BS to align more interference signals. Furthermore, it is shown that the sum-DoF is close to the DoF upper bound when the value of $M$ is large. This result implies that the proposed DSTIA method can asymptotically obtain the DoF upper bound when the number of antennas at each BS is large enough which will be very useful in the massive MIMO system.

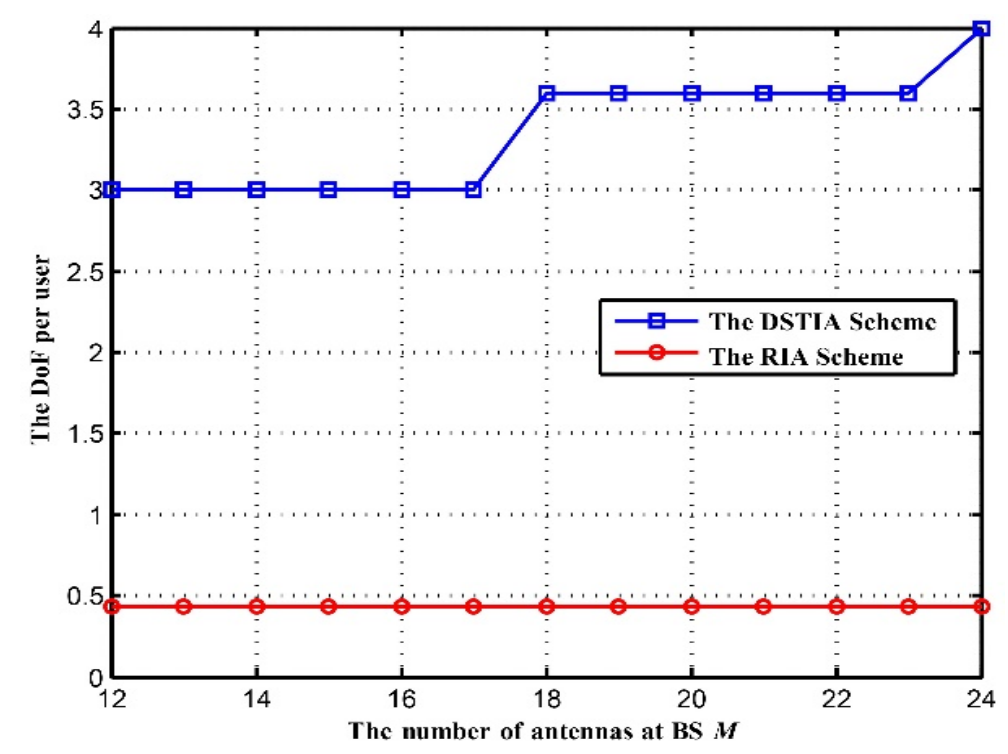

(a) System configuration $(L, N, K)=(2,6,2)$ 


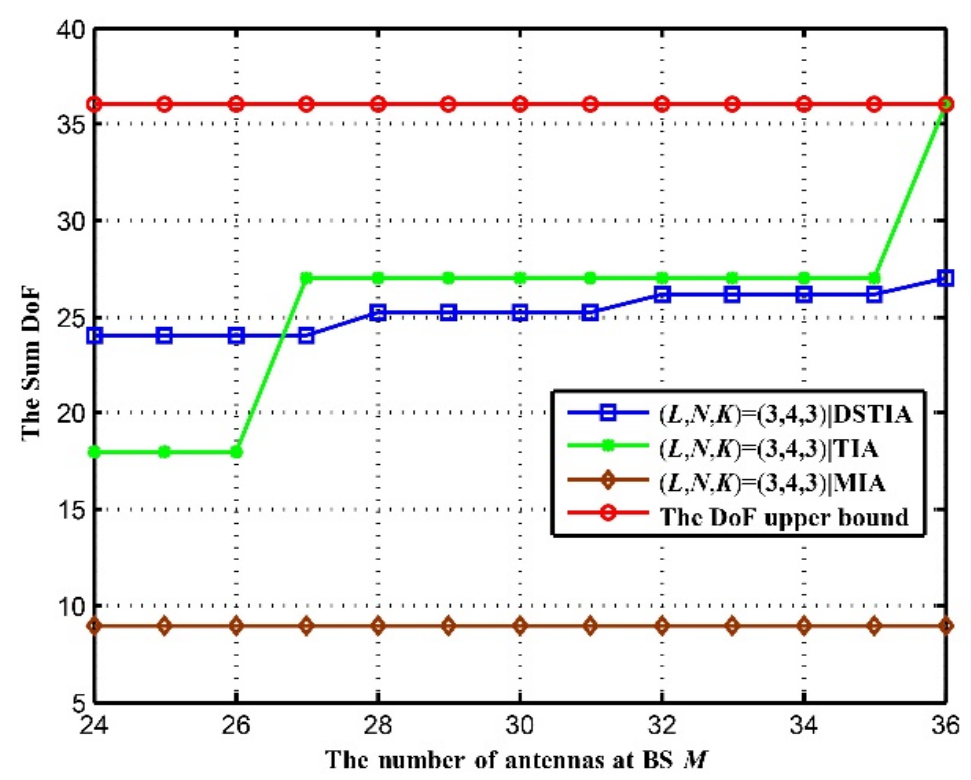

(b) System configuration $(L, N, K)=(3,4,3)$

Fig. 6. DoF comparison based on different schemes.

Fig. 6 plots the DoF results achieved by different schemes. In order to compare the DSTIA scheme with the RIA scheme in [21], the system configuration is set as two cells. The RIA scheme can utilize the delayed CSIT to obtain 3/7 DoF per user. In Fig. 6(a), it is assumed that $(L, N, K)=(2,6,2)$. It is shown that the obtained DoF of the proposed DSTIA approach is higher than the obtained DoF of the RIA scheme. In Fig. 6(b), we compare the proposed DSTIA scheme with the IA scheme by using global CSIT, i.e., the MAC-BC duality based on IA scheme (MIA) in [18] and the transceiver design based on IA (TIA) in [19]. In [18], the required system configuration is $M \geq(K(L-1)+1) \times d$ and $N \geq((K-1)(L-1)+1) \times d$, where $d$ denotes the number of interference-free data streams per user. When the values of $L$, $K, M$ and $N$ are given, the number of $d$ can be obtained directly. Similarly, the achievable DoF in [19] can be obtained. Although the TIA scheme can outperform the DSTIA and MIA scheme in the range of $M \in[27,36]$, the obtained DoF of the proposed DSTIA method is still higher than the MIA scheme in the range of $M \in[24,36]$ and the TIA scheme in the range of $M \in[24,26]$. The main reason is that the IA schemes with global CSIT cannot fully utilize signal spaces at BS to align more interference signals when the number of antennas at each user is small. However, the proposed scheme can take advantage of the antennas at each BS to transmit more interference-free data streams according to the design 
of ICI and IUI beamforming matrices with the current and outdated CSIT. This implies that the strict condition of global CSIT is not necessarily required in some system configurations and can be gradually degraded.

\section{Conclusion}

In this paper, we investigated the DoF of a multi-cell multi-user MIMO interference broadcast channel with non-cooperation distributed BS. The DSTIA scheme was proposed to eliminate the ICI and IUI signals by using the distributed moderately-delayed CSIT. We showed that the DSTIA scheme can obtain the achievable DoF and asymptotically achieve the DoF upper bound when the number of antennas at each BS is large enough. From the numerical simulation results, it was shown that the DSTIA approach can provide significant DoF gains over the conventional TDMA scheme as well as other IA schemes with delayed CSIT in literature. In addition, the DSTIA scheme can obtain higher DoFs than the IA schemes with global CSIT for certain antenna configurations. It is an interesting future topic to study the DoF of the full duplex multi-cell multi-user MIMO cellular interference channel.

\section{References}

[1] M. A. Maddah-Ali, A. S. Motahari and A. K. Khandani, "Communication over MIMO X channels: interference alignment, decomposition, and performance analysis," IEEE Transactions on Information Theory, vol.54, no.8, pp. 3457-3470, 2008.

Article (CrossRef Link).

[2] V. R. Cadambe and S. A. Jafar, "Interference alignment and the degrees of freedom of wireless X networks,” IEEE Transactions on Information Theory, vol. 55,no. 9, pp. 3893-3908, 2009.

Article (CrossRef Link).

[3] Maddah-Ali M A and Tse D, "Completely stale transmitter channel state information is still very useful,” IEEE Transactions on Information Theory, vol. 58, no. 7, pp:4418-4431, 2012.

Article (CrossRef Link).

[4] H. Maleki, S. A. Jafar, and S. Shamai, "Retrospective interference alignment over interference networks," IEEE Journal of Selected Topics in Signal Processing, vol. 6, no. 3, pp. 228-240, 2012. Article (CrossRef Link).

[5] A. Ghasemi, A. S. Motahari, and A. K. Khandani, "On the degrees of freedom of X channel with delayed CSIT,” in Proc. of IEEE Int.symp.inf.theory, vol.42, no.4, pp. 767-770, 2011.

Article (CrossRef Link).

[6] M. J. Abdoli, A. Ghasemi, and A. K. Khandani, "On the degrees of freedom of $K$-user SISO interference and X channels with delayed CSIT," IEEE Transactions on Information Theory, vol. 59, no. 10, pp. 6542-6561, 2013. Article (CrossRef Link).

[7] Yang S, Kobayashi M, Gesbert D, et al, "Degrees of freedom of time correlated MISO broadcast channel with delayed CSIT,” IEEE Transactions on Information Theory, vol. 59, no. 1, 
pp.315-328, 2012. Article (CrossRef Link).

[8] Lee N and Heath R W, "Space-time interference alignment and degree-of-freedom regions for the MISO broadcast channel with periodic CSI feedback," IEEE Transactions on Information Theory, vol. 60, no. 1, pp.515-528, 2013. Article (CrossRef Link).

[9] Lee N, Tandon R, Jr R W H, "Distributed space-time interference alignment with moderately delayed CSIT," IEEE Transactions on Wireless Communications, vol. 14, no. 2, pp.1048 - 1059, 2015. Article (CrossRef Link).

[10] Ying T, Feng W, Su W, et al, "On the degrees of freedom of MIMO X networks with non-cooperation transmitters," IEEE Transactions on Wireless Communications, vol. 16, no. 4, pp.2490-2504, 2017. Article (CrossRef Link).

[11] C. Suh and D. Tse, "Interference alignment for cellular networks," in Communication, Control, and Computing 46th Annual Allerton, pp. 1037-1044, 2008. Article (CrossRef Link).

[12] W. Shin, N. Lee, J.-B. Lim, C. Shin and K. Jang, "On the design of interference alignment scheme for two-cell MIMO interfering broadcast channels," IEEE Transactions on Wireless Communications, vol.10, no.2, pp. 437-442, 2011. Article (CrossRef Link).

[13] C. Suh, M. Ho and D. N. Tse, "Downlink interference alignment," IEEE Transactions on Communications, vol.59, no.9, pp. 2616-2626, 2011. Article (CrossRef Link).

[14] K. Lee, "Uplink interference alignment for two-cell MIMO interference channels," IEEE Transactions on Vehicular Technology, vol.62, no.2, pp. 1861-1865, 2013.

Article (CrossRef Link).

[15] H. J. Chou, C. C. Chou, J. M. Wu and R. Y. Chang, "On the achievable degrees of freedom of two-cell multiuser MIMO interference networks," IEEE Transactions on Communications, vol.62, no.8, pp. 2880-2893, 2014. Article (CrossRef Link).

[16] Y. Ma, J. Li, R. Chen and Q. Liu, "On feasibility of interference alignment for $L$-cell constant cellular interfering networks,” IEEE Communications Letters, vol.16, no.5, pp. 714-716, 2012. Article (CrossRef Link).

[17] Liu T, Yang C, "Genie tree and degrees of freedom of the symmetric MIMO interfering broadcast channel,” IEEE Transactions on Signal Processing, vol.64, no.22, pp.5914-5929, 2016. Article (CrossRef Link).

[18] Tang J, Lambotharan S, "Interference alignment techniques for MIMO multi-cell interfering broadcast channels,” IEEE Transactions on Communications, vol.61, no.1, pp.164-175, 2013. Article (CrossRef Link).

[19] Lee H H, Kim M J, Ko Y C, "Transceiver design based on interference alignment in MIMO interfering broadcast channels," IEEE Transactions on Wireless Communications, vol.13, no.11, pp.6474-6483, 2012. Article (CrossRef Link).

[20] Shin W, Lee J, "Retrospective interference alignment for the two-cell MIMO interfering multiple access channel," IEEE Transactions on Wireless Communications, vol.14, no.7, pp.3937-3947, 2015. Article (CrossRef Link). 
[21] Torrellas M, Agustin A, Vidal J, "Retrospective interference alignment for the MIMO interference broadcast channel," IEEE International Symposium on Information Theory, pp.1492-1496, 2015. Article (CrossRef Link).

[22] Ge X, Zi R, Wang H, et al, "Multi-User Massive MIMO Communication Systems Based on Irregular Antenna Arrays,” IEEE Transactions on Wireless Communications, vol.15, no.8, pp. 5287-5301, 2016. Article (CrossRef Link).

[23] Li C, Zhang S, Liu P, et al, "Overhearing Protocol Design Exploiting Intercell Interference in Cooperative Green Networks,” IEEE Transactions on Vehicular Technology, vol.65, no.1, pp. 441-446, 2016. Article (CrossRef Link).

[24] Li C, Wang J, Zheng F C, et al, "Overhearing-Based Co-Operation for Two-Cell Network With Asymmetric Uplink-Downlink Traffics," IEEE Transactions on Signal \& Information Processing Over Networks, vol.2, no.3, pp. 350-361, 2016. Article (CrossRef Link).

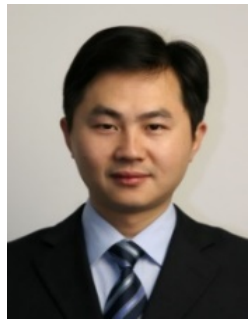

Hongbing Huang received his MS degree in electrical engineering from Wuhan University in 2003. He is currently working in State Grid Zhejiang Information \& Telecommunication Company. His research interests include electric power system communication, image processing technology.

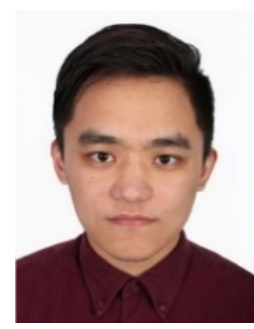

Junyi Liu received his MS degree in Zhejiang University in March 2014. He is an engineer in State Grid Zhejiang Electric Power Co.,Ltd. His research fields include network communication analysis and image processing.

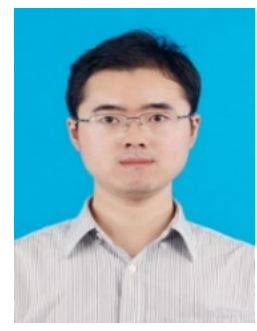

Yi Zhang received his MS degree in electrical engineering from University of Science and Technology of China in June 2010. His research fields include wireless network communication and fiber-optic communication. 


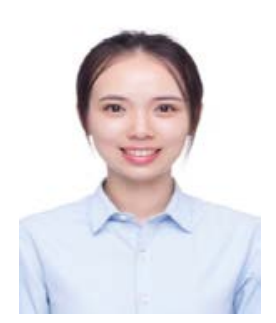

Qing Cai received her MS degree in communication and information system from Northeastern University in January 2017. She is an engineer in State Grid Zhejiang Electric Power Co.,Ltd. Her research fields include wireless optical network and routing algorithm.

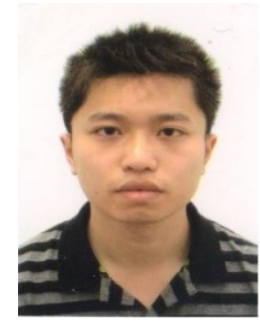

Bowei Zhang received his Ph.D degree from the College of Communication Engineering, Chongqing University (CQU), Chongqing, China in June 2017. His research interests include interference alignment, signal alignment and network coding in MIMO relay system.

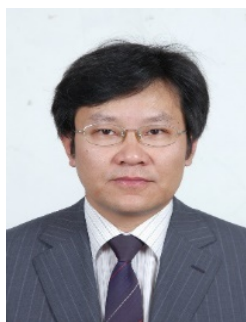

Wenjiang Feng received his Ph.D. degree in electrical engineering from Chongqing University in 2000. Currently, he is a professor at the college of communication engineering in Chongqing University. His research interests fall into the broad areas of communication theory, wireless communication. 